\title{
DESENVOLVIMENTO E AVALIAÇÃO DO USO DE APOSTILA VIRTUAL INTERATIVA PARA O ENSINO DA GEOESTATÍSTICA
}

DOI: 10.37702/2175-957X.COBENGE.2021.3443

PEDRO HENRIQUE ALVES CAMPOS - pedrocampos@demin.ufmg.br UFMG sao paulo 1091

30170-133 - Belo Horizonte - MG

Thalles Eduardo Cruz Morais - thalleseduardomorais@gmail.com Escola de Engenharia UFMG

Rua Doutor Rocha 691

33600-000 - Pedro Leopoldo - MG

Lucas Vieira Mendes - vieira1.lucass@gmail.com

UFMG

Rua José Basílio 260

30570-520 - Belo Horizonte - MG

Rayssa Victoria de Andrade - rayssavictoriadeandrade@gmail.com UFMG

Rua Santa Juliana 75

30550-220 - Belo Horizonte - MG

Resumo: A geoestatística é uma ciência que alia teoria e prática computacional, vertentes que devem ser contempladas no seu ensino. Atualmente, os docentes dessa disciplina enfrentam dois problemas: a falta de material didático adequado em língua portuguesa e a dependência de softwares para uso e prática na análise de dados. Como solução inovadora para tais dificuldades, desenvolveu-se duas apostilas virtuais interativas. Essas apostilas constituem-se de conteúdo teórico escrito em língua portuguesa e de códigos computacionais implementados para a análise dos dados. Assim, em um só ambiente, o aluno encontra a fonte teórica e pode também entender a prática, interagindo com o sistema e analisando criticamente os resultados provenientes de cada parâmetro de entrada diferente. Uma avaliação com dezessete alunos foi realizada e comprovou-se o potencial do uso das apostilas como ferramenta adicional no ensino e aprendizagem dessa ciência. 


\section{(C) COBENGE

Palavras-chave: Ensino de geoestatística. Apostila virtual interativa. Programação. Jupyter Notebook. Pluto Notebook. 


\section{DESENVOLVIMENTO E AVALIAÇÃO DO USO DE APOSTILA VIRTUAL INTERATIVA PARA O ENSINO DA GEOESTATÍSTICA}

\section{INTRODUÇÃO}

A geoestatística é o estudo estatístico de fenômenos naturais, caracterizados pela distribuição no espaço tridimensional de uma ou mais variáveis, denominadas "variáveis regionalizadas" (JOURNEL; HUIJBREGTS, 1978).

Desde a concepção da geoestatística nos anos 60 até hoje, vários livros de referência na área já foram publicados. Em sua maioria, caracterizam-se por possuírem uma grande quantidade de formalismo e notações matemáticas aparentemente complexas, que são de difícil compreensão para o aluno que está tendo o primeiro contato com essa ciência. Nesse contexto, alguns livros surgem como opções de bibliografias por adotarem uma linguagem mais acessível e notação mais simplificada, como por exemplo o livro $A n$ Introduction to Applied Geostatistics (ISAAKS; SRIVASTAVA, 1989). Entretanto, o estudante brasileiro se depara com um obstáculo adicional, que é a falta de referências didáticas disponíveis em língua portuguesa. Uma das poucas alternativas é o livro Geoestatística: Conceitos e Aplicações (YAMAMOTO; LANDIM, 2013).

A escassez de referências em língua portuguesa, traduzidas ou nativas, é um problema atual enfrentado pelos docentes de geoestatística no ensino superior em todo o Brasil. Enquanto alguns professores optam pela indicação de livros em língua inglesa, outros optam pelas poucas referências em português, por apostilas ou até mesmo pela não indicação de nenhuma referência. No ensino do tipo à distância ou remoto, esse problema é ainda mais grave, pois o material didático assume um papel ainda maior no processo de construção do conhecimento e de facilitador da aprendizagem (SILVA; SILVA, 2015).

Outra particularidade do ensino da geoestatística está no fato de que, se tratando de uma ciência aplicada à problemas práticos, é impossível dissociar teoria e prática, pois uma complementa a outra. $\mathrm{O}$ estudo apenas da parte teórica prejudica a aprendizagem e o entendimento holístico da geoestatística, sendo clara a necessidade de utilização de softwares específicos no estudo e análise dos fenômenos espaciais durante o ensino da disciplina.

O docente deve, portanto, escolher um software para utilização. Dentre as possibilidades, alguns são comerciais, enquanto outros são de uso público. Os comerciais possuem interface amigável, são constantemente atualizados e possuem suporte da empresa representante para seu uso. Entretanto, como desvantagem, apresentam um custo relativamente alto. Os softwares de uso público são gratuitos, mas a usabilidade geralmente é uma desvantagem, seja por falta de uma boa interface, por falta de atualizações ou por bugs. Não há, dessa forma, uma opção ideal.

Este trabalho apresenta o desenvolvimento e uso de duas apostilas virtuais interativas com o objetivo de atenuar os dois problemas previamente apresentados.

As apostilas possuem o conteúdo teórico formal atualizado da disciplina em língua portuguesa, podendo ser utilizadas pelos discentes como fonte de aprendizagem e estudo. São virtuais pois são acessadas a partir de um navegador de internet. São interativas, pois permitem a interação do usuário nas partes práticas da análise dos dados. Ao final de um semestre letivo, as apostilas foram apresentadas aos estudantes, que as avaliaram. 


\section{DESENVOLVIMENTO}

Três alunos de iniciação científica, auxiliados pelo professor orientador, desenvolveram duas apostilas virtuais interativas durante o período de um ano. Uma das apostilas foi desenvolvida no ambiente Jupyter Notebook utilizando a linguagem de programação Python 3, enquanto a segunda apostila interativa foi desenvolvida no ambiente Pluto, utilizando a linguagem Julia.

O propósito das duas apostilas é o mesmo: propiciar a aprendizagem teórica concomitantemente à prática da análise geoestatística de dados. Entretanto, por especificidades de cada ambiente de programação e da diferente disponibilidade de bibliotecas existentes, as apostilas se mostram esteticamente distintas e com pequenas diferenças quanto à análise feita.

O banco de dados utilizado como exemplo para análise nas duas apostilas é o banco de dados geoestatístico Walker Lake (ISAAKS; SRIVASTAVA, 1989). Outros bancos de dados podem ser importados e analisados nas apostilas, desde que os códigos sejam adaptados às suas especificidades.

Ao final do segundo semestre letivo de 2020, as duas apostilas foram apresentadas aos alunos da disciplina. Através de um formulário, dezessete alunos avaliaram a experiência do uso dessa ferramenta inovadora.

\subsection{Apostila interativa no Jupyter Notebook}

O ambiente Jupyter Notebook é acessado através do navegador de internet e é um documento virtual. Ele é organizado em células que podem ser configuradas como células do tipo markdown ou células de código.

No primeiro tipo, o conteúdo da célula é interpretado e formatado como um texto de informação, semelhante aos textos no software Microsoft Word. É possível formatar o conteúdo dessa célula de acordo com a finalidade, havendo inúmeras possibilidades de customização. Caso se deseje destacar o conteúdo, por exemplo, pode-se aplicar uma coloração ao fundo da célula, aumentar o tamanho da fonte, ou ainda deixar a escrita em negrito ou itálico. Pode-se também incluir imagens, equações, vídeos. A Figura 1 apresenta, como exemplo de customização, o início da apostila interativa, que contém imagem, título e informações iniciais. 
Figura 1 - Exemplo de customização de células markdown.

\section{DEMIN \\ DEPARTAMENTO DE ENGENHARIA DE MINAS}

\section{APOSTILA VIRTUAL INTERATIVA DE GEOESTATÍSTICA}

EMN 020 - Geoestatística

versão 1.0

Essa apostila virtual tem como objetivos ser uma fonte de conteúdo teórico de geostatística e demonstrar a sua aplicação na análise de dados geoespaciais.

O banco de dados apresentado como exemplo é o apresentado no livro An Introduction to Applied Geostatistics (1989). O workflow da análise consta de:

1. Análise exploratória dos dados

2. Modelagem da continuidade espacial através de variogramas

3. Estimativa espacial através de krigagem

Fonte: Elaborado pelo autor.

No segundo tipo, o conteúdo da célula é lido como um código de programação e, quando executado, gera um resultado que pode ser visualizado logo em sequência. A Figura 2 apresenta um exemplo de célula markdown seguido por célula de código com o resultado após sua execução. 
Figura 2 - Exemplo de organização célula markdown seguida por célula de código e seu resultado.

\author{
BOXPLOT
}

Um diagrama de caixa (Boxplot) é um gráfico de dados que consiste em uma reta que se prolonga do menor ao maior valor, e um retângulo construído com o primeiro quartil, a mediana e o terceiro quartil (Triola, 1999). Essa representação gráfica descreve simultaneamente diversas características importantes de um conjunto de dados, tais como tendências centrais, dispersão, desvio da simetria e identificação de outliers (valores extremos) (Montgomery; Runger, 2009)

- Execute o código abaixo para gerar o box-plot das variáveis $\mathrm{U}(\mathrm{ppm})$ e $\mathrm{V}(\%)$.

In [6]: \#BOX-PLOT DAS VARIÁVEIS V E U dO BANCO DE DADOS

plt. subplot(131)

dadosgeo.boxplot (column=[' $v(\%)$ '], figsize $=(8,5)$, grid=False)

plt.title( 'Box-plot da variável $\vee(\%)$ ")

plt.subplot (132)

dadosgeo.boxplot $\left(\right.$ column $=\left[{ }^{\prime} U(\mathrm{ppm})^{\prime}\right]$, figsize $=(8,5)$, grid=False $)$

plt.title('Box-plot da variável U(ppm)')

plt. subplots adjust (left $=0.0$, bottom $=0.0$, right $=3 . \theta$, top $=1.0$, wspace $=0.3$, hspace $=0.3$ )

plt.show()
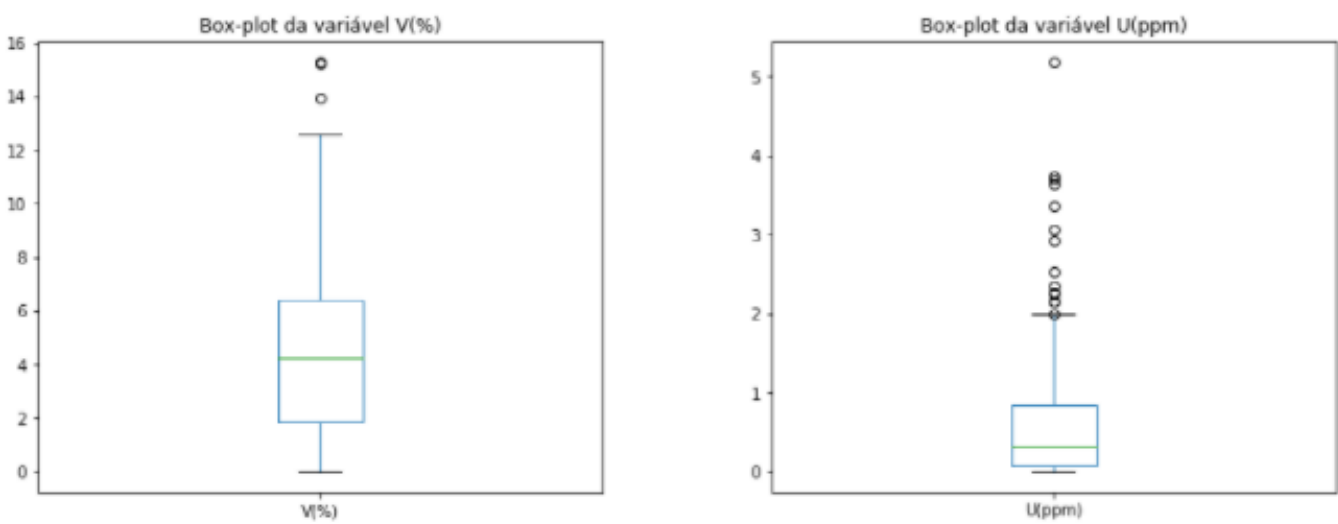

Fonte: Elaborado pelo autor.

Essa organização peculiar em células markdown e células de código é ideal para situações em que se deseja ter conteúdos informativos alternando com a execução de códigos e análises computacionais. Portanto, esse ambiente se mostra propício para o desenvolvimento de uma apostila que visa a aliar a informação teórica da disciplina com a parte prática da geoestatística.

Para a realização da parte prática, é necessária a utilização de diversas bibliotecas conhecidas nas ciências de dados, como o Pandas, MatplotLib, Numpy, Sklearn, dentre outras. Uma biblioteca é uma coleção de funções e métodos pré-existentes que executam ações específicas. Por exemplo, a biblioteca Pandas contém diversas funções relacionadas à manipulação e estruturação de dados. Assim, para importar dados em forma de tabela, não é necessário desenvolver um código novo, basta importar a referente função da biblioteca Pandas. A biblioteca Matplotlib, por sua vez, é uma coleção de funções para visualização de dados através de gráficos. Caso se deseje visualizar um histograma, é necessário apenas importar a função referente nessa biblioteca.

Assim como existem bibliotecas para manipulação de dados estruturados, visualização de dados, e cálculo numérico computacional, bibliotecas específicas para a análise geoestatística já foram desenvolvidas, como por exemplo o GeostatsPy. Além de conter funções necessárias à análise estatística, que são comuns à outras diversas bibliotecas, essa biblioteca também fornece funções necessárias à análise de dados geoespaciais, como a do cálculo do semivarigrama experimental, a de modelagem, e a do cálculo da krigagem. 
A disponibilidade de inúmeras bibliotecas com várias finalidades para a linguagem Python permite que várias ações possam ser executadas ao longo da apostila através de poucas linhas de código.

Atualmente em sua versão 1.0, a apostila virtual no Jupyter Notebook apresenta todos os procedimentos de uma análise geoestatística convencional já implementados, começando pela análise exploratória dos dados, passando pelo cálculo e modelagem dos semivariogramas direcionais, até o processo de estimativa por krigagem e validação. Não é necessário que o aluno modifique os códigos existentes ou que ele mesmo codifique, bastando apenas que ele execute os códigos.

Entretanto, o mais interessante na apostila virtual é que ela é também interativa. Parâmetros de entrada devem ser informados pelo usuário através do teclado. Ainda, alguns dos códigos foram escritos de forma que os parâmetros de entrada podem ser alimentados pelo arraste de sliders ou barras deslizantes, com possibilidade de visualização simultânea do resultado, conforme Figura 3.

Figura 3 - Exemplo de código no qual os parâmetros de entrada devem ser dados pelo arraste dos sliders e o resultado é visualizado simultaneamente no gráfico abaixo.

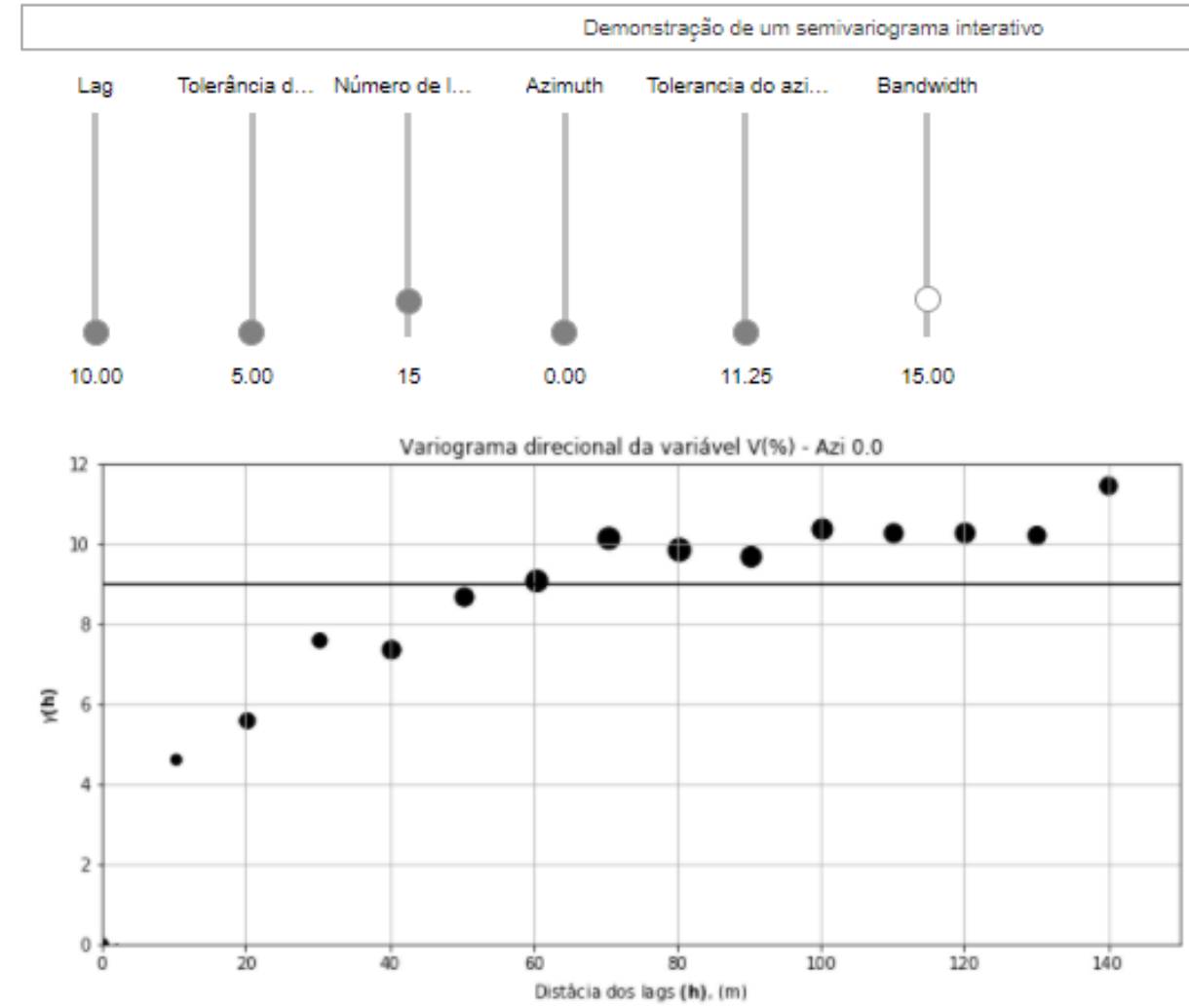

Fonte: Elaborado pelo autor.

Essa interatividade permite ao aluno entender na prática os efeitos e consequências dos parâmetros de entrada, auxiliando-o na tomada de decisão para a realização de uma estimativa.

\subsection{Apostila interativa no Pluto Notebook}

A apostila interativa no Pluto Notebook possui semelhanças e diferenças em relação ao Jupyter Notebook. 
Assim como o Jupyter, o Pluto também é organizado por células de código e de markdown. Entretanto, enquanto no Jupyter o usuário deve executar toda célula de código manualmente, no Pluto a execução de todas as células se dá automaticamente com a abertura do arquivo. Como consequência positiva, o Pluto permite ocultar a visualização das células de código, sendo apenas seus resultados visualizados. Uma vez que não é requerido do aluno a codificação, a apostila se apresenta mais agradável esteticamente e menos poluída visualmente, conforme Figura 4.

Figura 4 - Os códigos no Pluto são ocultados, de forma que apenas o conteúdo informativo e os resultados dos códigos sejam visíveis.

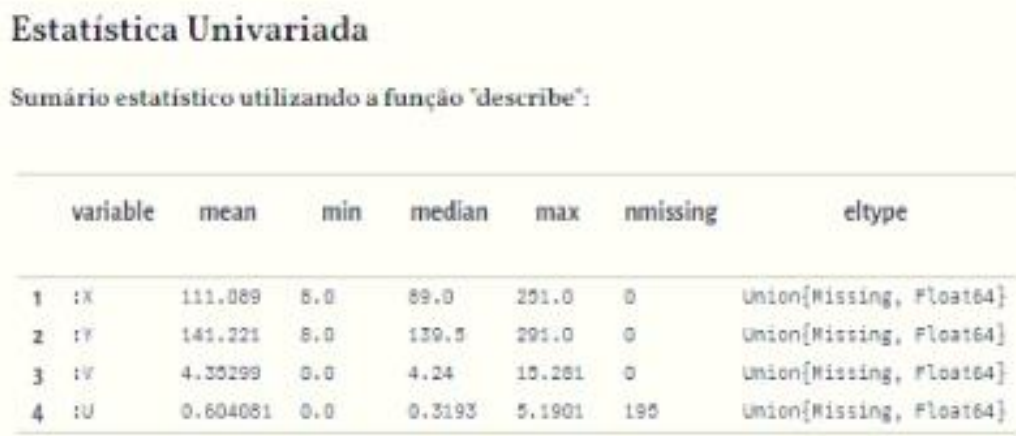

Restante do sumário estatistico:

\begin{tabular}{lllllll} 
& variable & DP & Q1 & median & Q3 & CoefVar \\
\hline 1 : V & 3.0 & 1.85 & 4.24 & 6.41 & nissing \\
2 tU & 0.77 & 0.08 & 0.32 & 0.84 & 1.27
\end{tabular}

Histogramas das variaveis $\mathrm{V} \in \mathrm{U}$ :
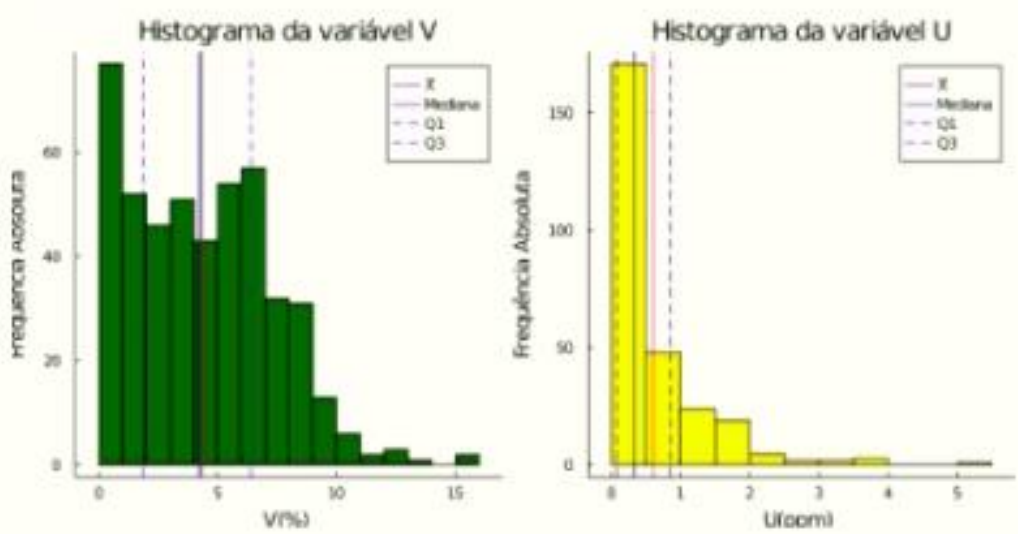

Fonte: Elaborado pelo autor.

Outra vantagem do Pluto é que as células de código apresentam interdependência. Isso significa que, ao modificar um parâmetro em uma célula, as células dependentes posteriores e anteriores são automaticamente atualizadas. Entretanto, essa mesma propriedade faz do Pluto um ambiente mais lento.

Outro ponto negativo do Pluto é a falta de variedade de bibliotecas existentes, devido a linguagem Julia ser mais recente e não tão tradicional quanto Python na ciência de dados. A única biblioteca de ferramentas geoestatísticas encontrada para o Pluto no momento é o Geostats.jl. Apesar disso, essa biblioteca apresenta funções geoestatísticas suficientes para a análise do banco de dados utilizado como demonstração. A Figura 5 mostra o 
resultado de um código para geração do variograma, com possibilidade de alteração dos parâmetros pelo usuário a partir das barras deslizantes, e a Figura 6 mostra o resultado da estimativa de uma região por krigagem e sua variância.

Figura 5 - Resultado do código do variograma. É possível alterar os parâmetros através das barras deslizantes.
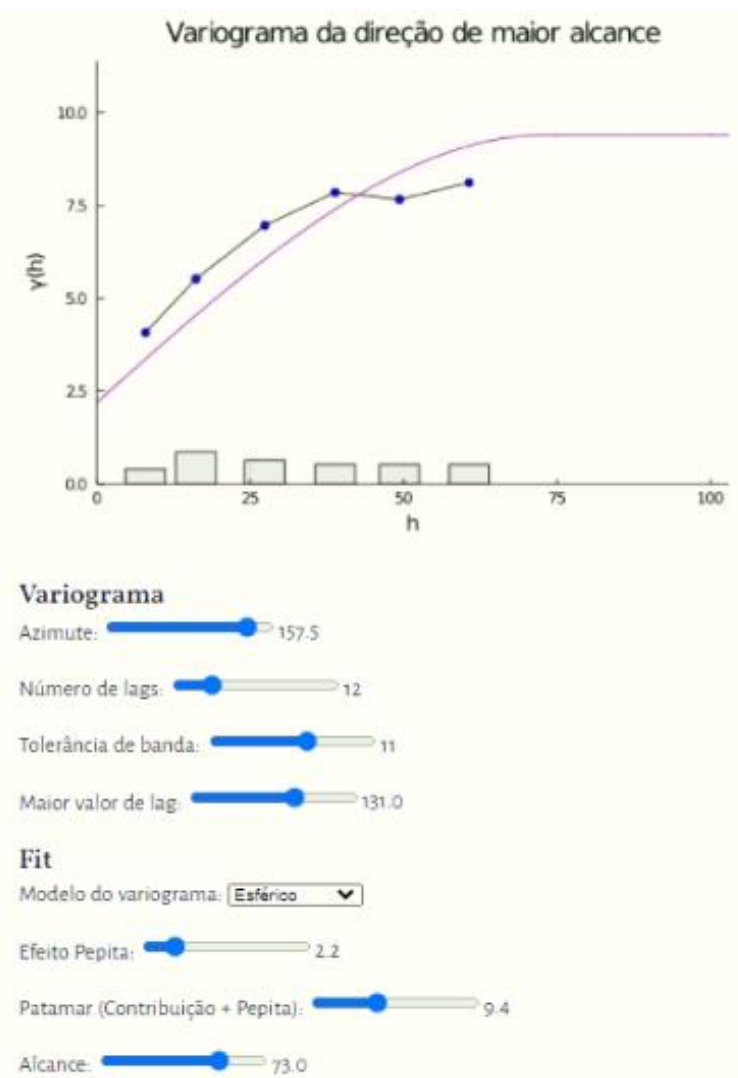

Fonte: Elaborado pelo autor.

Figura 6 - Resultado do código da krigagem.

Estimativa por Krigagem Ordinária:
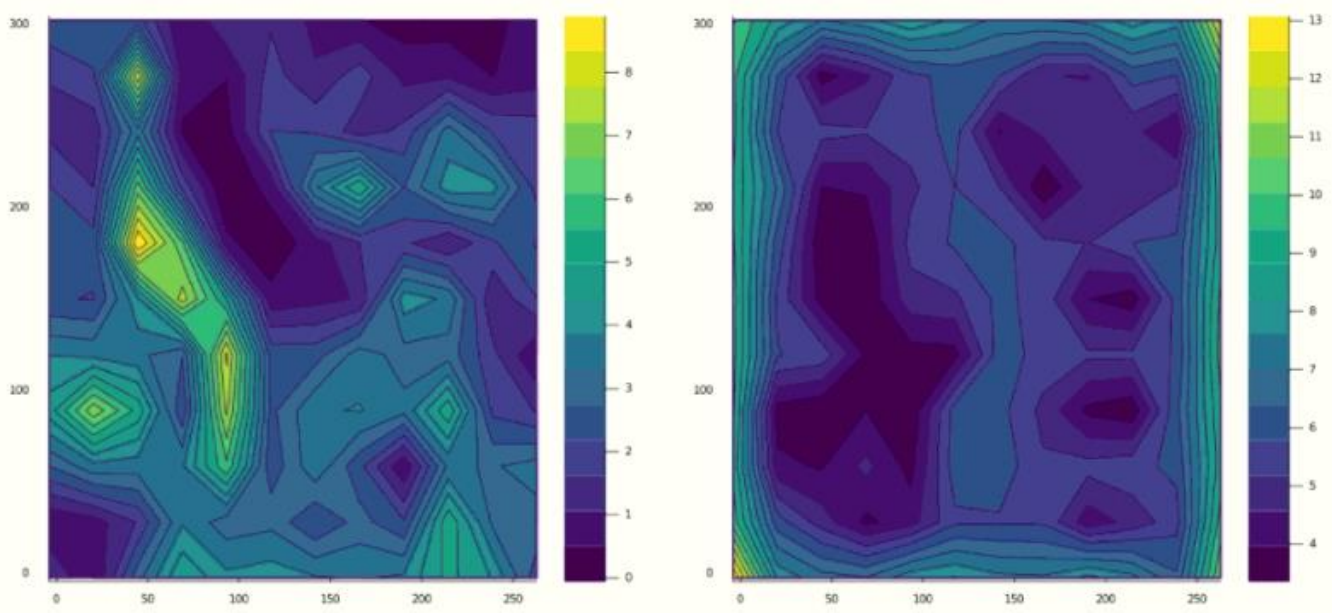

Fonte: Elaborado pelo autor. 


\section{$3 \quad$ RESULTADOS E DISCUSSÃO}

Para avaliar as potencialidades das apostilas como ferramenta de ensino da geoestatística, elas foram apresentadas aos alunos da disciplina no último dia de aula do período letivo. Esses alunos, por já terem absorvido o conteúdo e por terem tido experiência prévia com outras ferramentas de ensino, são capazes de melhor avaliar as qualidades e defeitos dessa nova ferramenta.

Incentivou-se os alunos para que lessem a parte teórica, executassem os códigos e que interagissem com o sistema, variando os parâmetros de entrada a fim de entender os impactos nos resultados. Por fim, um questionário de avaliação foi preenchido por dezessete alunos.

A Tabela 1 e a Tabela 2 mostram como os alunos avaliaram as dificuldades e as experiências durante o uso das apostilas.

Tabela 1 - Avaliação sobre dificuldades dos alunos no uso das apostilas

\begin{tabular}{|c|c|c|c|c|c|}
\hline & Nenhuma & Baixa & Intermediária & Alta & Muito alta \\
\hline $\begin{array}{c}\text { Dificuldade na } \\
\text { instalação das } \\
\text { apostilas }\end{array}$ & $29,4 \%$ & $47,1 \%$ & $11,8 \%$ & $5,9 \%$ & $5,9 \%$ \\
\hline $\begin{array}{c}\text { Dificuldade na } \\
\text { execução das } \\
\text { apostilas }\end{array}$ & $23,5 \%$ & $41,2 \%$ & $35,3 \%$ & $0,0 \%$ & $0,0 \%$ \\
\hline
\end{tabular}

Fonte: Elaborado pelo autor.

Tabela 2 - Avaliação sobre experiências do aluno durante o uso das apostilas

\begin{tabular}{|c|c|c|c|c|c|}
\hline & Péssima & Ruim & Média & Boa & Excelente \\
\hline $\begin{array}{c}\text { Experiência do uso } \\
\text { das apostilas para a } \\
\text { análise geoestatística } \\
\text { (parte prática) }\end{array}$ & $0,0 \%$ & $0,0 \%$ & $5,9 \%$ & $29,4 \%$ & $64,7 \%$ \\
\hline $\begin{array}{c}\text { Experiência do uso } \\
\text { das apostilas em } \\
\text { comparação ao } \\
\text { software convencional }\end{array}$ & $0,0 \%$ & $5,9 \%$ & $29,4 \%$ & $35,3 \%$ & $29,4 \%$ \\
\hline $\begin{array}{c}\text { Potencial das apostilas } \\
\text { como ferramenta de } \\
\text { ensino na disciplina }\end{array}$ & $0,0 \%$ & $0,0 \%$ & $0,0 \%$ & $17,6 \%$ & $82,4 \%$ \\
\hline
\end{tabular}

Fonte: Elaborado pelo autor.

Levantou-se também a opinião dos alunos sobre vantagens, desvantagens e outros comentários relevantes.

Sobre as vantagens, destaca-se: combinação da explicação teórica seguida da parte prática da análise, facilitando o entendimento dos procedimentos; organização da apostila de forma simples, intuitiva e linear, conforme o passo-a-passo de uma análise geoestatística tradicional; facilidade no uso e rapidez para análise de resultados a partir de diferentes parâmetros de entrada. 
Sobre as desvantagens, os aspectos mais comentados foram: rigidez nas possibilidades de análise, uma vez que o código já está implementado e só se pode variar os parâmetros de entrada; dependência de habilidades de programação para tornar as análises mais flexíveis; dependência da internet para instalação da apostila; visualização demasiadamente simplista dos resultados para uma análise a nível profissional.

Como comentários gerais, sobressai-se a opinião de que as apostilas possuem muito potencial para serem utilizadas como ferramentas de ensino. A maioria dos alunos opinam que as apostilam devem ser utilizadas em complemento ao software convencional já utilizado na disciplina, pois os pontos negativos de uma são pontos positivos da outra e vice-versa. Enquanto as apostilam fornecem uma boa visão geral de uma análise preliminar, o software possui mais flexibilidade para análises robustas, o que só é possível nas apostilas se o usuário possuir a capacidade de programação para editar os códigos implementados.

\section{CONSIDERAÇÕES FINAIS}

Os objetivos do desenvolvimento das apostilas foram cobrir, simultânea e integradamente, duas demandas existentes no ensino da geoestatística: um material fonte de informações teóricas em língua portuguesa e também um ambiente computacional de análise de dados gratuito e confiável.

A avaliação feita com os alunos mostrou que as apostilas possuem enorme potencial para serem utilizadas como ferramenta de ensino da disciplina em nível de graduação. A maioria dos alunos considerou a experiência do uso das apostilas na análise de dados como excelente. Em relação ao software convencional, as apostilas foram classificadas como boas. Deve ser dada atenção em relação às dificuldades nas suas instalações e execuções. Equilibrando suas vantagens e desvantagens, a opinião geral é de que as apostilas contribuirão para a melhoria do ensino e devem ser incluídas no programa da disciplina, mas não devem substituir o software convencional atualmente adotado.

É intenção dos autores que as apostilas sejam constantemente atualizadas e melhoradas. Contribui a isso o fato de que foram desenvolvidas em ambiente e linguagem de programação que são tendências tecnológicas atuais quando se trata de ciências de dados.

Destaca-se que não só o ensino da geoestatística pode se beneficiar com a adoção de tais apostilas virtuais interativas, mas também todas as disciplinas que possuem caráter numérico, estatístico e/ou computacional.

\section{REFERÊNCIAS}

ISAAKS, E.H.; SRIVASTAVA, R.M. An introduction to applied geostatistics. New York: Oxford University Press, 1989.

JOURNEL, A.G; HUIJBREGTS, C.J. Mining Geostatistics. London: Academic Press, 1978.

SILVA, Andreza Regina; SILVA, Daiana. Guia do professor conteudista. Florianópolis: IFSC, 2015.

YAMAMOTO, J.K; LANDIM, P. M. B. Geoestatística: Conceitos e Aplicações. $1^{\mathrm{a}}$ ed. São Paulo: Editora Oficina de Textos, 2013. 


\title{
DEVELOPMENT AND EVALUATION OF THE USE OF INTERACTIVE VIRTUAL COURSE NOTES FOR TEACHING GEOSTATISTICS
}

\begin{abstract}
Geostatistics is a science that combines theory and computational practice, aspects that must be considered in its teaching. Currently, teachers in this discipline face two problems: the lack of adequate didactic material in Portuguese and the dependence on software for use and practice in data analysis. As an innovative solution to such difficulties, two interactive virtual course notes were developed. These course notes consist of theoretical content written in Portuguese and computational codes implemented for data analysis. Thus, in a single environment, the student finds the theoretical source and can also understand the practice, interacting with the system and critically analyzing the results from each different input parameter. An assessment with seventeen students was carried out and the potential of using the virtual course notes as an additional tool in the teaching and learning of this science was proven.
\end{abstract}

Keywords: Geostatistics teaching. Interactive virtual course notes. Programming. Jupyter Notebook. Pluto Notebook. 